\title{
Triplane osteotomy combined with talar non-weight-bearing area autologous osteochondral transplantation for osteochondral lesions of the talus
}

\author{
Yan Zhang ${ }^{\dagger}$, Jing-qi Liang ${ }^{\dagger}$, Xiao-dong Wen, Pei-long Liu, Jun Lu and Hong-mou Zhao*
}

\begin{abstract}
Background: Traditional medial malleolar osteotomy combined with autologous osteochondral transplantation (AOT) is mostly used in the treatment of osteochondral lesions of the talus (OLTs), but with high osteotomy and donor site complications. We hypothesis a new triplane medial malleolar osteotomy combined with AOT from non-weightbearing area of the talus could be a promising choice for OLTS.

Methods: We reviewed all the symptomatic OLTs patients who received AOT with triplane osteotomy of the medial malleolus between September 2015 and December 2017 in our department. According to the inclusion and exclusion criteria, 23 patients (23 ankles), including 14 males and 9 females, were included in the study. The mean age was 35.6years. The mean size of the lesion area was $141.5 \mathrm{~mm}^{2}$. According Ferkel's classification, including 5 type I, 11 typella and 7 typellb. The visual analog scale (VAS) for pain during walking and the American Orthopaedic Foot and Ankle Society (AOFAS) ankle-hindfoot score were used for the pre- and postoperative evaluations. In addition, the incorporation of the grafts was assessed by computed tomography (CT).
\end{abstract}

Results: All patients had a minimum follow-up of 22 months, with an average of 37.1 months. The mean time from osteotomy to full weight-bearing activity was $8.1 \pm 2.3$ weeks (range, 5-12 weeks). The mean VAS score improved from $5.6 \pm 0.7$ preoperatively to $0.7 \pm 1.0$ postoperatively $(P<0.01)$. The AOFAS ankle-hindfoot score improved significantly in all domains $(P<0.01)$. Twenty-one patients returned to sport at their previous level, and 2 returned at a lower level compared with preinjury (mean return to play, 7.4 months). According to $C T$, the medial malleolus recovered in all patients, and the graft was incorporated well. One patient suffered from flexor hallucis longus tendon discomfort due to internal fixation screw irritation posteromedial to the ankle. The general complication rate was 4.3\% (1/23).

Conclusions: These results indicate that AOT combined with medial malleolus triplane osteotomy maybe a viable option for OLTs. Patients could perform weight-bearing exercise and return to sport as early as possible, with a lower rate of complications at the osteotomy site and donor site. However, the large sample well-designed prospective comparative studies are still needed.

Keywords: Talus, Medial malleolar osteotomy, Donor, Autograft, Osteochondral transplantation, Osteochondral lesions

*Correspondence: zhao_hongmou@hotmail.com

†Yan Zhang and Jing-qi Liang contributed equally to this work.

Department of Foot and Ankle Surgery, Honghui Hospital, Xi'an Jiaotong

University, 710054, Xi'an, Shaanxi Province, China

(c) The Author(s) 2022. Open Access This article is licensed under a Creative Commons Attribution 4.0 International License, which permits use, sharing, adaptation, distribution and reproduction in any medium or format, as long as you give appropriate credit to the original author(s) and the source, provide a link to the Creative Commons licence, and indicate if changes were made. The images or other third party material in this article are included in the article's Creative Commons licence, unless indicated otherwise in a credit line to the material. If material is not included in the article's Creative Commons licence and your intended use is not permitted by statutory regulation or exceeds the permitted use, you will need to obtain permission directly from the copyright holder. To view a copy of this licence, visit http://creativecommons.org/licenses/by/4.0/. The Creative Commons Public Domain Dedication waiver (http://creativeco mmons.org/publicdomain/zero/1.0/) applies to the data made available in this article, unless otherwise stated in a credit line to the data. 


\section{Background}

Osteochondral lesions of the talus (OLTs) are one of the most common sports injuries of the ankle joint. In addition, $50-73 \%$ of these lesions are associated with acute ankle injury events $[1,2]$, and sports participants are a susceptible population. OLTs is a kind of progressive disease, and may cause irreversible damage to the ankle joint and affect the quality of life with time delayed [3]. The treatment of symptomatic OLTs is difficult because of the poor blood supply of the talus. Conservative treatments for symptomatic OLTs include nonsteroidal drugs, sodium hyaluronate injection, physiotherapy, etc. However, a review reported that the success rate of conservative treatment was approximately $45 \%$ [4]. If nonoperative treatment fails, alternative surgical interventions include arthroscopic debridement and bone marrow stimulation (BMS), autologous osteochondral transplantation (AOT), allogeneic osteochondral transplantation, autologous or juvenile chondrocyte implantation, biological agents, and Hemi CAP ${ }^{\circledR}$ prosthetic implantation [5, 6]. Among these methods, arthroscopic procedures are mainly used for the cartilage lesions, and if the damage is deeper than $6 \mathrm{~mm}$, AOT is mostly used to solve the cartilage and bony problems [7].

Nguyen et al. [8] found that $78 \%$ of OLTs were located in the posteromedial area of the talus dome, i.e., areas IV-V and areas IV-VIII according to the "Division of the talus" [9] (Fig. 1). OLTs in these areas always require medial malleolar osteotomy to fully expose the lesions. However, a study [10] reported that the malunion rate after traditional medial malleolar oblique osteotomy was as high as $30 \%$, and approximately $60 \%$ of patients had residual pain and complaints at the osteotomy site, which seriously limited the outcomes of this procedure.

To reduce the complications related to traditional medial malleolar osteotomy, we designed a new triplane osteotomy method. In addition, the non-weight-bearing area of the ipsilateral talus was used as the donor site for AOT. We hypothesized that this new medial malleolar triplane osteotomy method would reduce osteotomyrelated complications, and AOT from the non-weightbearing area of the ipsilateral talus could achieve satisfactory clinical outcomes for OLTs.

\section{Methods}

The study was approved by the local institutional ethics committee. The authors retrospectively studied the clinical and radiological outcomes of OLTs patients who underwent medial malleolar triplane osteotomy with non-weight-bearing autografts between September 2015 and November 2017. The inclusion criteria were as follows: (1) adults older than 18 years of age; (2) a confirmed

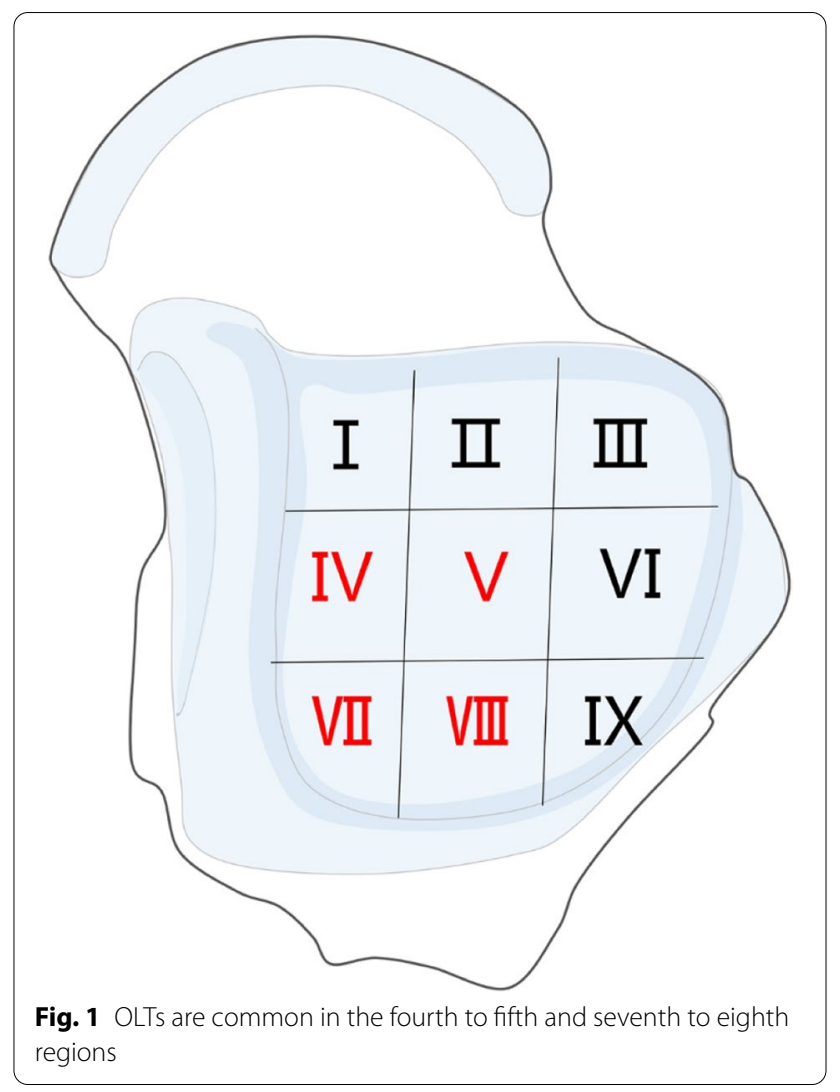

diagnosis of OLTs concomitant with a cystic depth greater than $6 \mathrm{~mm}$ by computed tomography (CT); (3) lesions located in areas IV, V, VI, VIII of the talus dome; (4) ineffective conservative treatment for more than 6 months; and (5) at least 22 months of follow-up. The exclusion criteria were as follows: (1) subchondral defect greater than $154 \mathrm{~mm}^{2}$ after debridement in the operation; (2) fracture malunion or malalignment of the lower limb; (3) ankle degeneration; (4) ankle joint instability; (5) a history of medial malleolar fracture or osteotomy; (6) revision surgery; and (7) poor general health condition.

A total of 51 OLT patients treated operatively were initially identified. According to the inclusion and exclusion criteria, 23 patients (14 males and 9 females) were included in the current study. The average age was $31.4 \pm 7.2$ (range, 19-46) years, and the mean body mass index (BMI) was $25.1 \pm 3.7$ (range, 19-33). Among the 23 ankles, surgery was performed on the left ankle in 13 patients (57\%) and on the right ankle in 10 patients (43\%), and the average duration of symptoms was $12.7 \pm 6.3$ (range, 6-33) months. The injury causes were ankle sprains (57\%) and fractures (13\%), and the injury cause could not be determined in 7 patients. For each patient, plain radiography, $\mathrm{CT}$, and magnetic resonance imaging (MRI) of the ankle joint were obtained, and the diagnosis 
of OLTs was confirmed. According to the "9-zone anatomic grid system", 19 lesions (83\%) were located in zone 4, 1 lesion (4\%) was located in zone 5, and 3 lesions (13\%) were located in zone 7 . In the current study, OLTs were classified by Ferkel's classification [11] based on CT scans. Among the OLTs, 5 OLTs were type I, 11 OLTs were typeIIa and 7 OLTs were typeIIb. The average area of the lesion was $113.2 \pm 28.1$ (range, $63.6-153.9$ ) $\mathrm{mm}^{2}$, and the mean depth of the cyst was $11.0 \pm 2.5$ (range, 7.0-16.0) $\mathrm{mm}$ after debridement during the operation (Table 1).

\section{Surgical technique}

All the operations were finished by the same surgeon (ZHM). Under general anesthesia, the patient was placed in a supine position with a tourniquet on the affected thigh. A $6 \mathrm{~cm}$ longitudinal curved incision was made from the anteromedial part of the inferior tibia and ended at the medial malleolar tip. The great saphenous vein was carefully located and protected. Medial malleolar triplane osteotomy method: First, a transverse osteotomy was performed in the anteromedial part of the ankle $2 \mathrm{~cm}$ above the tibial distal surface, with a depth of the anterior half of the tibia and a width of the medial half of the tibia. Second, a coronal osteotomy was performed along the midline of the medial malleolus. Third, a sagittal osteotomy was performed following the midline of the anterior tibia. Finally, the osteotomy block was carefully separated from the tibia with a thin osteotome. While the fragment was flipped downwards, the OLTs could be further visualized.

After the insertion of an appropriate recipient tube harvester, the unhealthy osteochondral plug was removed. The wall and base of the lesions were abraded and curetted down to the viable subchondral bone, and then a $1.0 \mathrm{~mm} \mathrm{~K}$-wire was used to make a "microfracture" in the lesions. After that, the articular cartilage was reconstructed by a cylindrical autologous osteochondral plug taken from the medial non-weight-bearing area of the ipsilateral talus. If the recipient cavity was too loose so that the graft was at risk of detachment, the gap was filled with autogenous cancellous tissue, which was harvested from the distal tibial section after the medial malleolar osteotomy until stabilization. The donor bone defect was filled with an autologous bone graft with periosteum from the tibia by the same

Table 1 Demographic data of OLT patients

\begin{tabular}{|c|c|c|c|c|c|c|c|c|c|c|}
\hline Cases & Sex & $\begin{array}{l}\text { Age } \\
\text { (yr) }\end{array}$ & BMI & Side & Etiology & $\begin{array}{l}\text { Duration } \\
\text { (mo) }\end{array}$ & Classification & Location & $\begin{array}{l}\text { Area of lesion } \\
\left(\mathrm{mm}^{2}\right)\end{array}$ & $\begin{array}{l}\text { Depth } \\
\text { of lesion } \\
(\mathrm{mm})\end{array}$ \\
\hline 1 & F & 29 & 25 & $\mathrm{R}$ & Sprain & 11 & lla & 7 & 78.5 & 7 \\
\hline 2 & $M$ & 32 & 26 & $L$ & Fracture & 20 & $\| a$ & 4 & 113.1 & 13 \\
\hline 3 & $\mathrm{~F}$ & 35 & 22 & $\mathrm{R}$ & Sprain & 12 & 1 & 4 & 132.7 & 8 \\
\hline 4 & $M$ & 36 & 30 & $L$ & Unknown & 8 & $\|$ lla & 4 & 78.5 & 16 \\
\hline 5 & $M$ & 44 & 25 & $L$ & Sprain & 19 & Ilb & 4 & 113.1 & 9 \\
\hline 6 & $M$ & 30 & 24 & $\mathrm{R}$ & Fracture & 12 & Ilb & 4 & 132.7 & 10 \\
\hline 7 & $\mathrm{~F}$ & 31 & 26 & $\mathrm{R}$ & Sprain & 6 & $\|$ lla & 4 & 153.9 & 9 \\
\hline 8 & M & 37 & 27 & $\mathrm{~L}$ & Unknown & 9 & Ilb & 7 & 132.7 & 11 \\
\hline 9 & M & 42 & 23 & $L$ & Unknown & 10 & $\| a$ & 4 & 113.1 & 13 \\
\hline 10 & $F$ & 31 & 29 & $L$ & Sprain & 7 & I & 4 & 113.1 & 11 \\
\hline 11 & $M$ & 27 & 28 & $\mathrm{R}$ & Sprain & 33 & Ila & 4 & 78.5 & 9 \\
\hline 12 & $M$ & 20 & 30 & $\mathrm{R}$ & Sprain & 21 & $\| \mathrm{a}$ & 4 & 153.9 & 14 \\
\hline 13 & $\mathrm{~F}$ & 31 & 21 & $L$ & Unknown & 9 & $\| \mathrm{b}$ & 4 & 63.6 & 10 \\
\hline 14 & $\mathrm{~F}$ & 29 & 23 & $L$ & Unknown & 11 & Ila & 4 & 113.1 & 9 \\
\hline 15 & $M$ & 19 & 27 & $\mathrm{R}$ & Sprain & 6 & 1 & 4 & 78.5 & 7 \\
\hline 16 & M & 27 & 21 & $L$ & Sprain & 8 & I & 5 & 78.5 & 9 \\
\hline 17 & F & 23 & 19 & $L$ & Unknown & 13 & $\| a$ & 4 & 153.9 & 13 \\
\hline 18 & M & 24 & 22 & $\mathrm{R}$ & Sprain & 12 & $\| \mathrm{a}$ & 4 & 132.7 & 10 \\
\hline 19 & $\mathrm{~F}$ & 41 & 33 & $L$ & Sprain & 9 & $\| \mathrm{b}$ & 4 & 132.7 & 14 \\
\hline 20 & M & 30 & 24 & $L$ & Unknown & 10 & $\| \mathrm{b}$ & 7 & 78.5 & 11 \\
\hline 21 & M & 27 & 22 & $L$ & Sprain & 14 & $\| a$ & 4 & 113.1 & 15 \\
\hline 22 & $\mathrm{~F}$ & 46 & 30 & $\mathrm{R}$ & Fracture & 21 & 1 & 4 & 132.7 & 11 \\
\hline 23 & $M$ & 32 & 20 & R & Sprain & 12 & $\| \mathrm{b}$ & 4 & 132.7 & 13 \\
\hline
\end{tabular}


recipient tube harvester. The periosteum was flush with the surrounding cartilage surface. Especially note that when the column was harvesting from the medial side of distal tibia, the periosteum was protected to ensure that it does not separate from the cortical bone.

Finally, the medial malleolar osteotomy site was fixed with 3 screws. The surgical diagram was seen in Figs. 2, and the operation detail was seen in Figs. 3 and 4.

\section{Postoperative care}

The ankle joints were wrapped in a bandage for 3 weeks. Patients were encouraged to begin passive range-ofmotion exercises for the ankle joint on the 3rd day after the operation The patients were initially restricted to non-weight-bearing activities, and in the absence of rehabilitation, they wore a short leg cast for protection. The patients wore removable walking boots and started partial weight-bearing activities at the 4th week post operation. Full weight-bearing activities began when X-rays
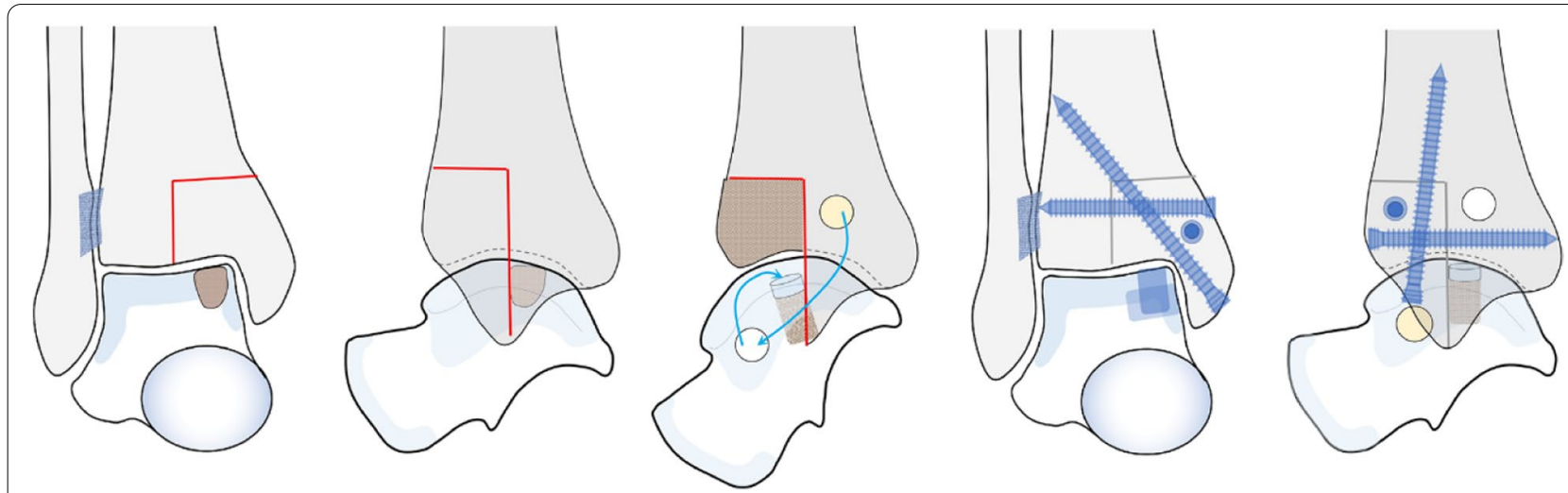

Fig. 2 A-B: medial malleolar triplane osteotomy; C: AOT for OLTs from a non-weight-bearing area, and the donor defect was filled with a bone graft with periosteum from the tibia; D-E: medial malleolar osteotomy fixed with screws

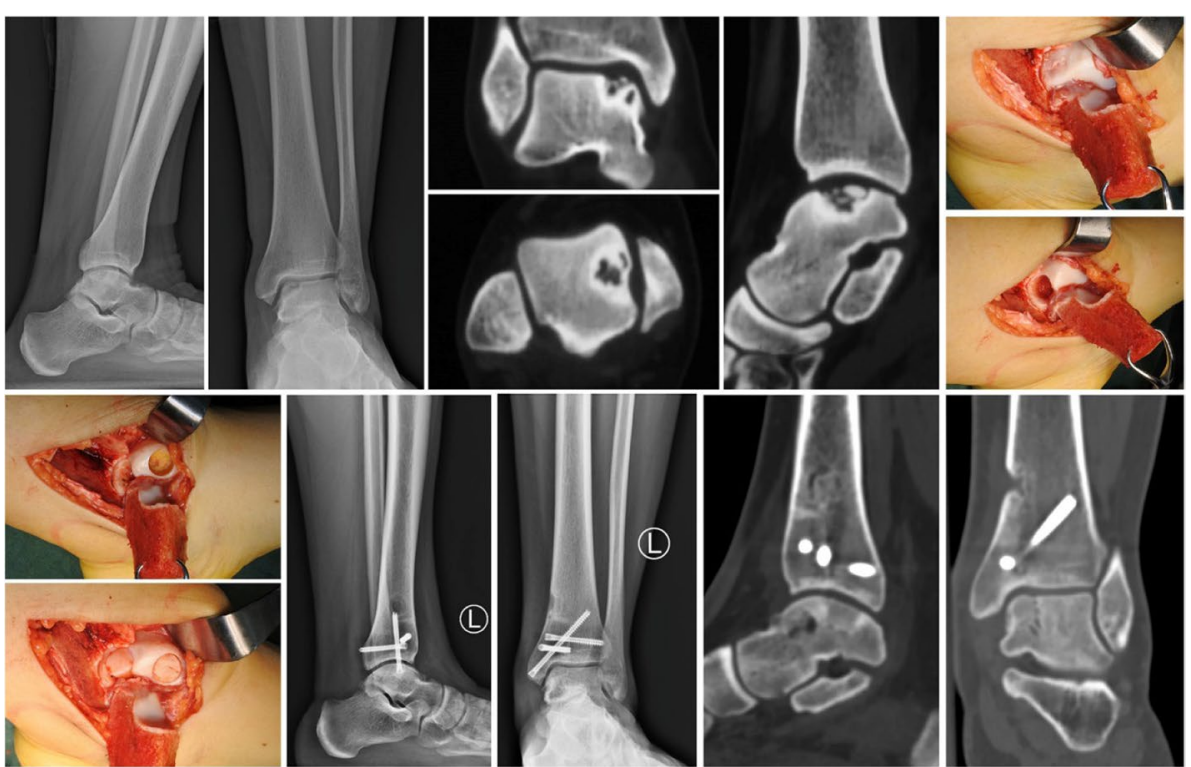

Fig. 3 Case1, female, 31y, osteochondral lesions of left talus. A-E: preoperative imaging (X - ray and CT) supported the diagnosis; F-G: the lesion was exposed through the medial malleolus triplane osteotomy and debrided; $\mathbf{H}-\mathbf{I}$ : AOT for OLTs from a non-weight-bearing area, and the donor defect was filled with a bone graft with periosteum from the tibia; J-M: at 37-month follow-up, imaging showed that the grafts had fused with the original bone in the talus, and the medial malleolus had achieved anatomic union 

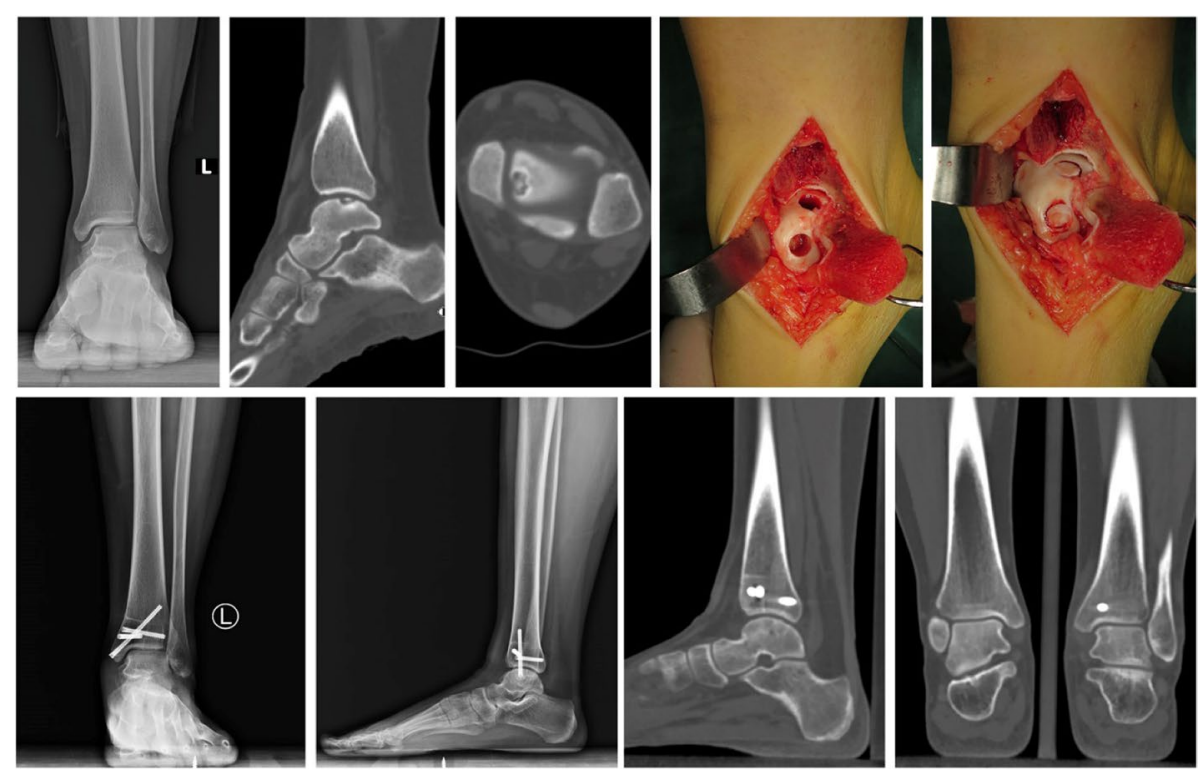

Fig. 4 Case2, female, 41y, osteochondral lesions of left talus. A-C: preoperative imaging ( $\mathrm{X}$ - ray and CT) supported the diagnosis; D-E: the lesion was exposed through the medial malleolus triplane osteotomy and AOT for OLTs from a non-weight-bearing area, and the donor defect was filled with a bone graft with periosteum from the tibia; F-I: at 1.5-year follow-up, imaging showed that the grafts had fused with the original bone in the talus, and the medial malleolus had achieved anatomic union

showed callus formation, which is evidence of bone healing at the site of the medial malleolar osteotomy.

\section{Clinical and radiographic evaluation}

The clinical outcome was assessed using the American Orthopedic Foot and Ankle Society ankle-hindfoot (AOFAS-AH) score and the visual analog scale (VAS) score. To show the actual impact of pain on patients' daily activities, we evaluated the VAS score after $1 \mathrm{~km}$ of walking. The AOFAS-AH and VAS scores were obtained both preoperatively and at the final follow-up. In addition, we recorded the operation time, postoperative complications, time of full weight-bearing activities and the ROM of the affected ankle joint at the final follow-up. X-rays and $\mathrm{CT}$ scans were acquired for all patients to assess the reduction and healing of the medial malleolar osteotomy site, the union of grafts in the talus and the position and length of internal screws. X-ray is necessary in every follow-up after operation. After 3 months postoperatively, CT was used for evaluation at each follow-up. The malunion of medial malleolus osteotomy was defined as medial malleolus incongruence with measurable proximal or medial displacement. Degenerative changes (including narrowing of joint space, formation of osteophyte and increase of subchondral bone density) of ankle were observed based on X-ray. All the included clinical and radiological measurements on were performed by two observers independently (WXD and LPL), and the mean of the two observers was used as the result.

\section{Statistical analysis}

IBM SPSS 21.0 software (SPSS Inc., Chicago, Illinois) was used for statistical analysis. The Shapiro-Wilk test was used to test whether the data were normally distributed: if the data were normally distributed, Student's t-test was conducted to evaluate changes in the AOFAS-AH score and VAS score; if the data were not normally distributed, a rank sum test was used. The level of statistical significance was set at $\alpha=0.05$.

\section{Results}

The mean follow-up time was $37.1 \pm 7.3$ (range, 22-49) months. The mean time of operation was $69.4 \pm 12.6$ (range, 51-97) minutes. All medical malleolar osteotomy sites healed anatomically at a mean of $8.1 \pm 2.3$ (range, 5-12) weeks, with neither malunion nor nonunion. All the grafts in the talus were well fused according to CT scans until the final follow-up. One patient suffered from flexor hallucis longus tendon discomfort due to internal fixation screw irritation posteromedial to the ankle. The symptoms disappeared after the removal of the screw at 6 months post operation. In the current study, the general complication rate was $4.3 \%(1 / 23)$. At the last follow-up, the mean angles of affected ankle plantar flexion and dorsiflexion were $44.7^{\circ}$ and $12.1^{\circ}$, respectively. All 
patients were satisfied with the pain relief. The mean VAS score improved from $5.6 \pm 0.7$ (range, $4-7$ ) to $0.7 \pm 1.0$ (range, 0-3). The mean AOFAS-AH score improved from $56.0 \pm 9.5$ (range, 40-78) before the operation to $93.8 \pm 6.6$ (range, 83-100) at the final follow-up $(p<0.01)$ (Table 2). In addition, typical cases are shown below (Figs. 3 and 4).

\section{Discussion}

The most important finding of this study is that medial malleolar triplane osteotomy with AOT from the nonweight-bearing area of the talus for OLTs achieved significant pain reduction and satisfactory ankle function with few complications after a mean follow-up of more than 3 years.

Throughout evolution, to satisfy the needs of flexible ankle joint movement in human upright walking and activities, the talus, located in the center of the ankle, has gradually acquired a unique anatomy in that the majority of its surface (approximately $60 \%$ ) is covered with articular cartilage [12], and there is little soft tissue (including nutritional arteries) attachment in the body of the talus. On the other hand, the vessel spreading in the talus has failed to achieve a rich blood supply, forming a "watershed" of blood supply between single areas [13]. This anatomical limitation has been implicated in the high risk of posttraumatic osteonecrosis and OLTs [5, 13]. Nondisplaced or mild symptomatic OLTs are often initially treated with a nonoperative approach. Conservative treatments of OLTs include activity modification, protected weight-bearing, rehabilitation, bracing, and nonsteroidal anti-inflammatory drugs. Clinically, a systematic review by Verhagen [14] et al. showed that approximately $45 \%$ of patients with OLTs reported successful outcomes with conservative treatment. It is worth noting that the success of nonsurgical treatment for OLTs is mostly in the pediatric population, but it is limited in the adult population, which may be attributable to the relatively active repair ability of articular hyaline cartilage in young people. At present, there is no unified standard for surgical indications for OLTs. It is generally believed that patients with small OLTs less than $15 \mathrm{~mm}$ in diameter or

Table 2 Functional outcomes of AOT for OLTs

\begin{tabular}{|c|c|c|c|c|c|}
\hline \multirow[t]{2}{*}{ Time } & \multicolumn{4}{|c|}{ AOFAS-AH score } & \multirow{2}{*}{$\begin{array}{l}\text { VAS score } \\
(1 \mathrm{~km})\end{array}$} \\
\hline & Pain & Function & Alignment & Total & \\
\hline $\begin{array}{l}\text { Preop- } \\
\text { eration }\end{array}$ & $21.7 \pm 3.9$ & $24.3 \pm 6.3$ & 10 & $56.0 \pm 9.5$ & $5.6 \pm 0.7$ \\
\hline $\begin{array}{l}\text { Postop- } \\
\text { eration }\end{array}$ & $35.7 \pm 5.1$ & $48.1 \pm 1.9$ & 10 & $93.8 \pm 6.6$ & $0.7 \pm 1.0$ \\
\hline $\mathrm{t}$ & -13.371 & -22.463 & - & -24.835 & 33.631 \\
\hline$p$ & 0.000 & 0.000 & - & 0.000 & 0.000 \\
\hline
\end{tabular}

less than $150 \mathrm{~mm}^{2}$ in area can obtain satisfactory clinical effects through BMS [5, 15], whereas patients with large OLTs or failed bone marrow stimulation should choose replacement procedures $[5,16]$. A previous study [17] showed that the main pathway of BMS for OLTs is through a series of inflammatory reactions and cell differentiation to form new scar tissue (mainly type I collagen) to repair the talus defect area. However, some studies [18, 19] that used MRI to evaluate the imaging results of BMS in the treatment of OLTs found that the lesions could not be completely filled, the quality of scar tissue involved in the repair of articular surface was poor, and it was difficult to achieve complete fusion with primary cartilage. This may be related to the fact that the osteocytes under the cartilage of OLTs are not filled with bone after BMS treatment, resulting in the lack of effective physical support and nutritional supply for the surface tissue. Therefore, we believe that the limitation of osteochondral transplantation is not simply determined by the size of the lesion. In other words, even if the area of an articular cartilage lesion is relatively large $\left(>150 \mathrm{~mm}^{2}\right)$ and there is no cystic change or the cyst is very shallow, the use of BMS can also obtain sufficient scar tissue filling and repair. In contrast, for OLTs with significant cystic lesions, even if the area of cartilage lesions is not large, the OLT is still suitable for osteochondral transplantation because of the continuous effective physical support and sustainable nutritional supply for the repaired surface tissue through the approach. In the current study, we took a cystic depth of OLTs greater than $6 \mathrm{~mm}$ as an indication for AOT. On the other hand, patients with OLT areas greater than $144 \mathrm{~mm}^{2}$ were excluded to avoid the adverse effect of using multiple bone columns for transplantation.

AOT was mainly previously applied to the surgical treatment of osteochondral lesions of the knee joint [20]. The operation is to repair the defect of the articular cartilage surface by transplanting the autogenous bonecartilage column into the lesions. At the same time, the subchondral bone in the graft fully fills the cavity and finally integrates with the original bone, providing necessary and continuous nutritional and physical support for the cartilage. The goal of AOT is to implant a graft that is similar in both mechanical and biological properties to that of the patient's native hyaline cartilage. Several retrospective case series have demonstrated positive results with AOT [8, 16, 21]. Imhoff et al. [22] observed significant long-term improvements in mean AOFAS, VAS, and Tegner activity scores in 26 patients. At a mean followup time of 7 years, 18 patients indicated they were very satisfied, 4 satisfied, 3 neutral, and 1 moderately unsatisfied with the procedure. Shimozono et al. [21] systematically reviewed 11 mid-term clinical follow-up studies on AOT treatment for OLTs, including 500 cases in ankle 
joints, with an average follow-up time of 62.8 months. They found that the average AOFAS ankle and hind foot scores increased from 55.1 preoperatively to 86.2 at the last follow-up, with an excellent rate of $87 \%$. This is similar to the clinical results of the current study reported by us. Autologous grafts are most commonly harvested from the ipsilateral knee, specifically from the lateral femoral condyle or the intercondylar notch. The primary concern with osteochondral autograft transfer is donor site morbidity, as reported by several studies [23]. Shimozono et al. [21] also found that the general incidence of complications after AOT was $10.6 \%$, the most important of which was related to the donor site, and the incidence of donor site morbidity was approximately $3.6 \%$. Fraser et al. [24] reported 40 OLT patients in whom the lateral femoral condyle of the same knee joint was used as the donor site for AOT. After an average follow-up of 24 months, they found that approximately $12.5 \%$ of the patients had discomfort symptoms at the donor site, including a painful knee joint and chondromalacia patella during high-intensity exercise. In our study, only 1 case of posterior tibial tendon discomfort caused by the use of too many internal fixation screws after AOT was found. The incidence of complications was $4.3 \%$, which was significantly lower than that reported in previous literature, and there were no donor-site-related complications. This may be related to the selection of the vertical surface of the talus as the donor site. This surgery did not increase the operation time and avoided potential discomfort at the donor site. Also, the osteotomy area may be relative bigger than the oblique osteotomy, but do not increase the bleeding of osteotomy site, and the larger and vertical osteotomy may increase stability and bony union. On the other hand, we used the medial cortical bone of the distal tibia to backfill the talus donor site. The autogenous bone had a strong healing ability, and there was no risk of allogeneic infection. The last follow-up CT scan showed that all the donor-site defects achieved good healing, which also reduced the damage to the stress structure of the talus and the potential risk factors for complications.

The development of arthroscopy technology provided a good choice for minimally invasive treatment of OLTs. However, arthroscopic procedures are mainly used for the cartilage lesions, including debridement, microfracture and BMS. If the damage is deeper than $6 \mathrm{~mm}$, AOT is mostly used to solve the cartilage and bony problems [7]. In this study, the location of OLTs was region IV in 19 patients, region V in 1 patient, and region VII in 3 patients; thus, all the lesions were mainly concentrated in the posterior medial region of the talus where the OLTs usually needed medial malleolar osteotomy for exposure and operation. In general, all kinds of medial malleolar osteotomies not only facilitate the exposure of and operation at the posterior medial area of the talus but also bring additional damage to the medial sensitive area of the ankle joint and the risk of remaining discomfort. Kreuz et al. [25] believed that the total adverse effects of OLT may occur in 3 stages: (1) in the short term, the osteotomy itself can damage adjacent important anatomical structures, such as the posterior tibial tendon, tibial nerve and posterior tibial artery; (2) in the mid-term, the malunion or nonunion caused by poor reduction or fixation of medial malleolus can cause adverse effects; and (3) in the long term, the accelerated degeneration of local articular cartilage can lead to ankle arthritis. Bull et al. [10] reported that the rate of healing-induced deformity after internal malleolar biplane chevron osteotomy was up to $30 \%$. After 2.4 years of follow-up, it was found that approximately $24 \%$ of patients had swelling at the osteotomy site, and the residual rate of pain in the internal malleolus was up to $60 \%$. The main reasons were related to poor reduction and internal fixation failure after osteotomy. In the current study, we designed a new medial malleolus osteotomy procedure, a three-plane osteotomy of the medial malleolus, which has the following advantages: (1) By adding the coronal osteotomy of the medial malleolus on the biplane ladder osteotomy, the trauma caused by the osteotomy of the medial malleolus is reduced, the bleeding is reduced, and the adhesion of the posterior medial tendon of the ankle is avoided. (2) By retaining the integrity of the posterior medial bone of the medial malleolus, the risk of injury to important anatomical structures such as the posterior medial neurovascular tendon caused by complete osteotomy of the medial malleolus, such as oblique osteotomy, is avoided. (3) After osteotomy in this way, the osteotomy section at the proximal end of the tibia presents a three-dimensional square concave shape, which is convenient to achieve the complete anastomosis of three planes (the sagittal plane, coronal plane, and horizontal plane) under the direct vision when the distal medial malleolus is restored. In addition, the medial malleolus has high stability after reduction, and reduction is not easily lost during fixation. (4) When hollow screws are used to fix the inner malleolus, vertical compression and firm fixation of screws on three sides is allowed, permitting early functional exercise and weight-bearing activities. In the current study, with the triplane osteotomy method we used, malunion or nonunion of the medial malleolus was not observed, and there was no obvious sign of joint degeneration at the medial malleolar osteotomy site. The average time from osteotomy to full weight-bearing activity was 8.1 weeks, earlier than the medial malleolus healing time reported in the literature $[10,26,27]$. During the last follow-up, the ROM of the affected ankle joint was $12.1^{\circ}$ dorsiflexion and $44.7^{\circ}$ plantar flexion, and the degree of activity was 
basically restored to the preoperative level, without obvious ankle joint or tendon adhesion, which may be related to the early active rehabilitation training that we allowed.

There were a few limitations of the present study. First, this was a retrospective study and with all the inherent limitations of a retrospective study. Such as we did not measure the preoperative ROM of ankle joint. Second, this study included a limited number of patients to further conclude the complication or failure relative factors. Third, the follow-up time was relatively short, and the long-term clinical outcomes of this procedure is still unclear, especially the degenerative changes of ankle joint. And, this study did not compare the technique with other medial malleolar osteotomy procedures as a control group, and warrant further investigation.

\section{Conclusions}

These results indicate that AOT combined with medial malleolus triplane osteotomy maybe a viable option for OLTs. Despite the risk of secondary talus injury and internal fixation irritation, the clinical effect and imaging evaluation results at a short follow-up was favorable. The use of the vertical non-weight-bearing area of the talus as the transplantation donor area could reduce the risk of additional surgical injury. Triplane osteotomy of the medial malleolus could reduce the time of osteotomy and trauma, and it was convenient for anatomical reduction and strong internal fixation. However, due to the lack of comparative study with current treatment options, for both the medial malleolus osteotomy and the osteochondral lesion treatments, the advantages of the technique remain indeterminacy. So, the large sample well-designed prospective comparative studies are still needed in the future.

\section{Abbreviations \\ AOT: Autologous osteochondral transplantation; OLTs: Osteochondral lesions of the talus; BMI: Body mass index; CT: Computed tomography; AOFAS-AH: the American Orthopedic Foot and Ankle Society ankle-hindfoot; VAS: Visual analog scale; ROM: Range of motion.}

\section{Acknowledgements}

We are grateful to all the study participants for their contributions. We appreciate the entire data collection team.

\section{Authors' contributions}

ZY, LJQ and ZHM designed the study, analyzed the data, and wrote the manuscript. ZHM performed all the operations, and ZY, LJQ, WXD participated as surgical assistant. WXD and LPL collected the data, follow up of patients and helped in writing the manuscript. $L J$ participated in the design of the study and analyzed the data. All authors read and approved the final manuscript.

\section{Funding}

This study was supported by the China Postdoctoral Science Foundation funded project (2017 M613178), and Key R \& D plan of Shaanxi Province (2021SF-025).

\section{Availability of data and materials}

The data of this study were real and were performed in SPSS 21.0 software (SPSS Inc., Chicago, Illinois). The statistical results of the data are presented in this main paper. The images of case examples are depicted in this research article. All of the data are available in contact with the correspondence author.

\section{Declarations}

\section{Ethics approval and consent to participate}

All procedures performed in the current study involving human participants were in accordance with the ethical standards of the institutional and/or national research committee and with the 1964 Declaration of Helsinki and its later amendments or comparable ethical standards. For the triplane medial malleolar osteotomy, the new procedure is already approved for clinical use at our institution. We have obtained informed consent to participate from the participants. The consent and study plan was approved by the Ethics Committee of Honghui Hospital (202003002).

\section{Consent for publication}

Not applicable.

\section{Competing interests}

The authors declared no potential conflicts of interest with respect to the research, authorship, and/or publication of this article.

Received: 25 July 2021 Accepted: 19 January 2022

Published online: 22 January 2022

\section{References}

1. Prado MP, Kennedy JG, Raduan F, Nery C. Diagnosis and treatment of osteochondral lesions of the ankle: current concepts. Rev Bras Ortop. 2016;51(5):489-500.

2. Leontaritis N, Hinojosa L, Panchbhavi VK. Arthroscopically detected intraarticular lesions associated with acute ankle fractures. J Bone Joint Surg Am. 2009;91(2):333-9.

3. D’Ambrosi R, Maccario C, Serra N, Ursino C, Usuelli FG. Relationship between symptomatic osteochondral lesions of the talus and quality of life, body mass index, age, size and anatomic location. Foot Ankle Surg. 2018;24(4):365-72.

4. Zengerink M, Szerb I, Hangody L, Dopirak RM, Ferkel RD, van Dijk CN. Current concepts: treatment of osteochondral ankle defects. Foot Ankle Clin. 2006;11(2):331-59.

5. Looze CA, Capo J, Ryan MK, Begly JP, Chapman C, Swanson D, et al. Evaluation and management of osteochondral lesions of the talus. Cartilage. 2017;8(1):19-30.

6. Ebskov LB, Andersen KH, Rasmussen PB, Johansen JK, Benyahia M. Midterm results after treatment of complex talus osteochondral defects with HemiCAP implantation. Foot Ankle Surg. 2020;26(4):384-90.

7. Ferkel RD, Hommen JP. Arthroscopy of the ankle and foot. In: Coughlin MJ, Mann RA, Saltzman CL, editors. Surgery of the foot and ankle. 8th ed. Philadelphia: Elsevier; 2007. p. 1641-726.

8. Nguyen A, Ramasamy A, Walsh M, McMenemy L, Calder JDF. Autologous osteochondral transplantation for large osteochondral lesions of the talus is a viable option in an athletic population. Am J Sports Med. 2019;47(14):3429-35.

9. Elias I, Zoga AC, Morrison WB, Besser MP, Schweitzer ME, Raikin SM. Osteochondral lesions of the talus: localization and morphologic data from 424 patients using a novel anatomical grid scheme. Foot Ankle Int. 2007;28(2):154-61.

10. Bull PE, Berlet GC, Canini C, Hyer CF. Rate of malunion following bi-plane chevron medial malleolar osteotomy. Foot Ankle Int. 2016;37(6):620-6.

11. Ferkel RD, Flannigan BD, Elkins BS. Magnetic resonance imaging of the foot and ankle: correlation of normal anatomy with pathologic conditions. Foot Ankle. 1991;11(5):289-305.

12. Rachel JS, Nirmal CT. Surgical treatment of talus fractures. Orthop Clin North Am. 2013:44(4):521-8. 
13. Lomax A, Miller RJ, Fogg QA, Madeley NJ, Kumar CS. Quantitative assessment of the subchondral vascularity of the talar dome: a cadaveric study. Foot Ankle Surg. 2014;20(1):57-60.

14. Verhagen RAW, Struijs PAA, Bossuyt PMM, van Dijk CN. Systematic review of treatment strategies for osteochondral defects of the talar dome. Foot Ankle Clin. 2003:8(2):233-42.

15. Gianakos AL, Yasui Y, Hannon CP, Kennedy JG. Current management of talar osteochondral lesions. World J Orthop. 2017;8(1):12-20.

16. Park KH, Hwang Y, Han SH. Primary versus secondary osteochondral autograft transplantation for the treatment of large osteochondral lesions of the talus. Am J Sports Med. 2018;46(6):1389-96.

17. Bae DK, Yoon KH, Song SJ. Cartilage healing after microfracture in osteoarthritic knees. Arthroscopy. 2006;22(4):367-74.

18. Becher C, Zühlke C, Plaas C, Ewig M, Calliess T, Stukenborg-Colsman C et al. T2-mapping at $3 \mathrm{~T}$ after microfracture in the treatment of osteochondral defects of the talus at an average follow-up of 8 years. Knee Surg Sports Traumatol Arthrosc. 2015;23(8):2406-12.

19. Kuni B, Schmitt $H$, Chloridis D, Ludwig K. Clinical and MRI results after microfracture of osteochondral lesions of the talus. Arch Orthop Trauma Surg. 2012;132(12):1765-71.

20. Sherman SL, Thyssen E, Nuelle CW. Osteochondral autologous transplantation. Clin Sports Med. 2017;36(3):489-500.

21. Shimozono Y, Hurley ET, Myerson CL, Kennedy JG. Good clinical and functional outcomes at mid-term following autologous osteochondral transplantation for osteochondral lesions of the talus. Knee Surg Sports Traumatol Arthrosc. 2018;26(10):3055-62.

22. Imhoff AB, Paul J, Ottinger B, Wörtler K, Lämmle L, Spang J, et al. Osteochondral transplantation of the talus: long-term clinical and magnetic resonance imaging evaluation. Am J Sports Med. 2011;39(7):1487-93.

23. Shimozono Y, Seow D, Yasui Y, Fields K, Kennedy JG. Knee-to-talus donorsite morbidity following autologous osteochondral transplantation: a meta-analysis with best-case and worst-case analysis. Clin Orthop Relat Res. 2019;477(8):1915-31.

24. Fraser EJ, Savage-Elliott I, Yasui Y, Ackermann J, Watson G, Ross KA, et al. Clinical and MRI donor site outcomes following autologous osteochondral transplantation for talar osteochondral lesions. Foot Ankle Int. 2016;37(9):968-76.

25. Kreuz PC, Steinwachs M, Edlich M, Kaiser T, Mika J, Lahm A, et al. The anterior approach for the treatment of posterior osteochondral lesions of the talus: comparison of different surgical techniques. Arch Orthop Trauma Surg. 2006;126(4):241-6.

26. Leumann A, Horisberger $M$, Buettner $O$, Mueller-Gerbl M, Valderrabano V. Medial malleolar osteotomy for the treatment of talar osteochondral lesions: anatomical and morbidity considerations. Knee Surg Sports Traumatol Arthrosc. 2016;24(7):2133-9.

27. Sadlik B, Kolodziej L, Puszkarz M, Laprus H, Mojzesz M, Whyte GP. Surgical repair of osteochondral lesions of the talus using biologic inlay osteochondral reconstruction: clinical outcomes after treatment using a medial malleolar osteotomy approach compared to an arthroscopicallyassisted approach. Foot Ankle Surg. 2019;25(4):449-56.

\section{Publisher's Note}

Springer Nature remains neutral with regard to jurisdictional claims in published maps and institutional affiliations.

Ready to submit your research? Choose BMC and benefit from:

- fast, convenient online submission

- thorough peer review by experienced researchers in your field

- rapid publication on acceptance

- support for research data, including large and complex data types

- gold Open Access which fosters wider collaboration and increased citations

- maximum visibility for your research: over $100 \mathrm{M}$ website views per year

At BMC, research is always in progress.

Learn more biomedcentral.com/submissions 